\title{
Solvability Criterion for the Cauchy Problem for the Theory of Elasticity
}

\section{E. NiYOZOV}

Department of Mechanics and Mathematics, Samarkand State, University, Uzbekistan

*Corresponding Author: I. E. NIYozov, Department of Mechanics and Mathematics, Samarkand State, University, Uzbekistan

Abstract: In this paper, we consider problem of analytical continuation of a solution of the system equations of the thermoelasticity in bounded domain from its values and values of its strains on a part of the boundary of this domain, i.e., the Cauchy problem and we give a criterion for solvability of the Cauchy problem.

Keywords: The Cauchy problem; system theory of elasticity; elliptic system, ill-posed problem, Carleman matrix, regularization.

\section{INTRODUCTION}

In this paper, we consider problem of analytical continuation of a solution of the system equations of the thermo elasticity in bounded domain from its values and values of its strains on a part of the boundary of this domain, i.e., the Cauchy problem and we give a criterion for solvability of the Cauchy problem.

Let $x=\left(x_{1}, x_{2}, x_{3}\right)$ and $y=\left(y_{1}, y_{2}, y_{3}\right)$ be points of the 3 -dimensional Euclidean space $R^{3}, D$ be a bounded simply connected domain in $R^{3}$ with piecewise-smooth boundary consisting of a piece $\Sigma$ of the plane $y_{3}=0$ and a smooth surface $S$ lying in the half-space $y_{3}>0$.

Suppose $U(x)=\left(u_{1}(x), u_{2}(x), u_{3}(x), u_{4}(x)\right)$ is a vector function satisfies the following system of equations of the thermo elasticity in $D[1]$ :

$B\left(\partial_{x}, \omega\right) U(x)=0$,

where

$B\left(\partial_{x}, \omega\right)=\llbracket B_{k j}\left(\partial_{x}, \omega\right) \rrbracket_{4 \times 4}$

and

$B_{k j}\left(\partial_{x}, \omega\right)=\delta_{k j}\left(\mu \Delta+\rho \omega^{2}\right)+(\lambda+\mu) \frac{\partial^{2}}{\partial x_{k} \partial x_{j}}, k, j=1,2,3$,

$B_{k 4}\left(\partial_{x}, \omega\right)=-\gamma \frac{\partial}{\partial x_{k}}, \quad k=1,2,3$

$B_{4 j}\left(\partial_{x}, \omega\right)=-i \omega \eta \frac{\partial}{\partial x_{j}}, \quad j=1,2,3$,

$B_{44}\left(\partial_{x}, \omega\right)=\Delta+\frac{i \omega}{\theta}$,

$\delta_{k j}$ - is the Kronecker delta, $i=\sqrt{-1}, \omega-$ is the frequency of oscillation and $\lambda, \mu, \rho, \theta$ are its coefficients which characterizing medium, satisfying the conditions

$\mu>0, \quad 3 \lambda+2 \mu>0, \quad \rho>0, \quad \theta>0, \quad \frac{\gamma}{\eta}>0$.

System (1.1) can be written in the form: 
$\left\{\begin{array}{r}\mu \Delta u+(\lambda+\mu) \operatorname{grad} \operatorname{div} u-\gamma \operatorname{grad} v+\rho \omega^{2} u=0 \\ \Delta v+\frac{i \omega}{\theta} v+i \omega \eta \operatorname{div} u=0,\end{array}\right.$

where $U(x)=\left(u_{1}(x), u_{2}(x), u_{3}(x), u_{4}(x)\right)=(u(x), v(x)), u(x)=\left(u_{1}(x), u_{2}(x), u_{3}(x)\right)$.

Statement of the problem. Let $f=\left(f_{1}, f_{2}, f_{3}, f_{4}\right)^{T} \in\left[C^{1}(S)\right]^{4}, g=\left(g_{1}, g_{2}, g_{3}, g_{4}\right)^{T} \in[C(S)]^{4}$ be given vector-functions. It requires to find (if possible) a vector-function $U(x) \in\left[C^{1}(D \cup S) \cap\right.$ C2(D) 4 such that

$\left\{\begin{array}{c}B\left(\partial_{x}, \omega\right) U(x)=0 \text { in } D, \\ U(y)=f(y), y \in S, \\ R\left(\partial_{y}, n(y)\right) U(y)=g(y), \quad y \in S,\end{array}\right.$

where $R\left(\partial_{y}, n(y)\right)$ - is the stress operator, i.e.,

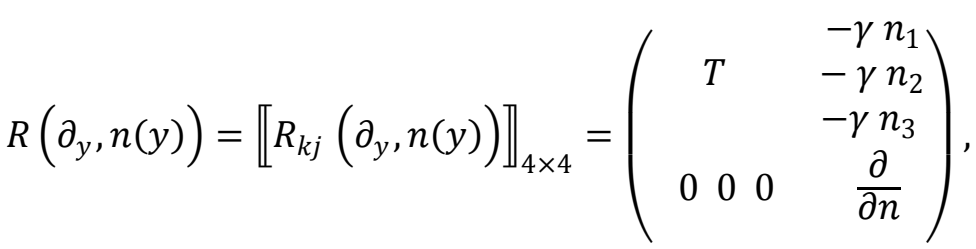

$T=T\left(\partial_{y}, n(y)\right)=\llbracket T_{k j}\left(\partial_{y}, n(y)\right) \rrbracket_{3 \times 3}$,

$T_{k j}\left(\partial_{y}, n(y)\right)=\lambda n_{k}(y) \frac{\partial}{\partial y_{j}}+\mu n_{j}(y) \frac{\partial}{\partial y_{k}}+(\lambda+\mu) \frac{\partial}{\partial n(y)}, \quad k, j=1,2,3$,

$n(x)=\left(n_{1}(x), n_{2}(x), n_{3}(x)\right)-$ is the unit outward normal vector on $\partial D$ at a point $y$.

Here $\left[C^{k}(S)\right]^{4},(k=0,1,2, \ldots)$ stands for the vector space of all 4-vector valued functions whose components are $k$ times continuously differentiable on a set $D \subset R^{3}$.

It is known that the system (1.2) is elliptic and problem (1.3) has no more than one solution. However, it is ill-posed, i.e. 1) not for any data there exists a solution; 2) solution do not depend continuously on the Cauchy data on $S$ (see, for example, [2]).Therefore, solvability conditions can not be described in terms of continuous linear functional.

In this paper we will apply the integral representation's method to obtain solvability conditions and a formula for solution of the problem.

\section{Construction of the Carleman Matrix and ApProximate Solution For the Cap TYPE DOMAIN FOR THE CAP TYPE DOMAIN}

It is well known that any regular solution $U(x)$ of the system (1.1) is specified by the formula [1]

$$
\begin{aligned}
2 U(x) & =\int_{\partial D}\left(\Psi(x-y, \omega)\left\{R\left(\partial_{y}, n(y)\right) U(y)\right\}-\right. \\
& \left.-\left\{\tilde{R}\left(\partial_{y}, n(y)\right) \widetilde{\Psi}(x-y, \omega)\right\}^{*} U(y)\right) d s_{y}, \quad x \in D,
\end{aligned}
$$

where the symbol $\{\cdot\}^{*}-$ means the operation of transposition, $\Psi(x-y, \omega)$ is the matrix of the fundamental solutions for the system of equations of steady-state oscillations of the thermoelasticity: given by

$$
\begin{aligned}
& \Psi(x, \omega)=\llbracket \Psi_{k j}(x, \omega) \rrbracket_{4 \times 4}, \\
& \Psi_{k j}(x, \omega)=\sum_{l=1}^{3}\left[\left(1-\delta_{k 4}\right)\left(1-\delta_{j 4}\right)\left(\frac{\delta_{k j}}{2 \pi \mu} \delta_{3 l}-\alpha_{l} \frac{\partial^{2}}{\partial x_{k} \partial x_{j}}\right)+\right. \\
& \left.+\beta_{l}\left(i \omega \eta\left(1-\delta_{j 4}\right) \delta_{k 4} \frac{\partial}{\partial x_{j}}-\gamma\left(1-\delta_{k 4}\right) \delta_{j 4} \frac{\partial}{\partial x_{k}}\right)+\delta_{k 4} \delta_{j 4} \gamma_{l}\right] \frac{\exp \left(i \lambda_{l}|x|\right)}{|x|},
\end{aligned}
$$


Where

$\alpha_{l}=\frac{(-1)^{l}\left(1-i \omega \theta^{-1} \lambda_{l}^{-2}\right)\left(\delta_{1 l}+\delta_{2 l}\right)}{2 \pi(\lambda+2 \mu)\left(\lambda_{2}^{2}-\lambda_{1}^{2}\right)}-\frac{\delta_{3 l}}{2 \pi \rho \omega^{2}}, l=1,2,3 ; \quad \sum_{l=1}^{3} \alpha_{l}=0$,

$\beta_{l}=\frac{(-1)^{l}\left(\delta_{1 l}+\delta_{2 l}\right)}{2 \pi(\lambda+2 \mu)\left(\lambda_{2}^{2}-\lambda_{1}^{2}\right)}, \quad l=1,2,3 ; \quad \sum_{l=1}^{3} \beta_{l}=0$,

$\gamma_{l}=\frac{(-1)^{l}\left(\lambda_{l}^{2}-k_{1}^{2}\right)\left(\delta_{1 l}+\delta_{2 l}\right)}{2 \pi\left(\lambda_{2}^{2}-\lambda_{1}^{2}\right)}, l=1,2,3 ; \sum_{l=1}^{3} \gamma_{l}=0$,

$k_{1}^{2}=\rho \omega^{2}(\lambda+2 \mu)^{-1}, \lambda_{3}^{2}=\frac{\rho \omega^{2}}{\mu}$

$\lambda_{1}^{2}$ and $\lambda_{2}^{2}$ are defined from the equation

$\lambda_{1}^{2}+\lambda_{2}^{2}=\frac{i \omega}{\theta}+\frac{i \omega \gamma \eta}{\lambda+2 \mu}+k_{1}^{2}, \quad \lambda_{1}^{2} \lambda_{2}^{2}=\frac{i \omega}{\theta} k_{1}^{2}$,

where $\lambda_{1}^{2} \neq \lambda_{2}^{2}$,

$\widetilde{\Psi}(x, \omega)=\llbracket \widetilde{\Psi}_{k j}(x, \omega) \rrbracket_{4 \times 4}, \quad \widetilde{\Psi}_{k j}(x, \omega)=\Psi_{k j}(-x, \omega)$

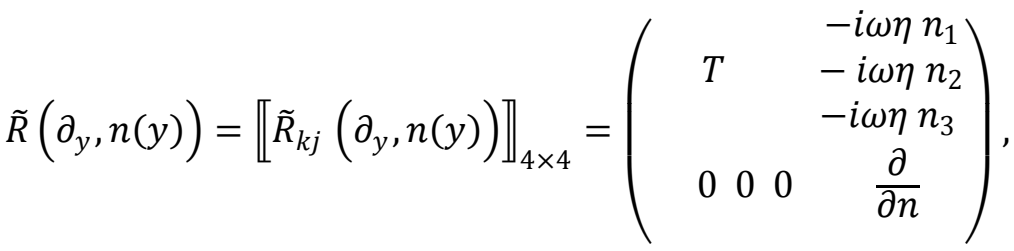

Definition. By the Carleman matrix of the problem (1.1), (1.3) we mean an $4 \times 4$ matrix $\Pi(y, x, \omega, \sigma)$ depending on the two points $y, x$ and a positive numerical number parameter $\sigma$ satisfying the following two conditions:

1) $\Pi(y, x, \omega, \sigma)=\Psi(x-y, \omega)+G(x-y, \sigma)$,

where the matrix $G(x-y, \sigma)$ satisfies system (1.1) with respect to the variable $y$ on $D$, and $\Psi(x-$ $y, \omega$ is a matrix of the fundamental solutions of system (1.1);

2) $\int_{\partial D \backslash S}\left(|\Pi(y, x, \omega, \sigma)|+\left|R\left(\partial_{y}, n(y)\right) \Pi(y, x, \omega, \sigma)\right|\right) d s_{y} \leq \varepsilon(\sigma)$,

where $(\sigma) \rightarrow 0$, as $\sigma \rightarrow \infty$; here $|\Pi|$ is the Euclidean norm of the matrix $\Pi=\llbracket \Pi_{k j} \rrbracket_{4 \times 4} \quad$ i.e.,

$|\Pi|^{2}=\left(\sum_{k, j=1}^{4} \Pi_{k j}^{2}\right)$

In particular,

$|U|^{2}=\left(\sum_{k=1}^{4} U_{k}^{2}\right)$

From the definition of Carleman matrix and from the Greens formulas it follows that

Theorem2.1. Any regular solution $U(x)$ of system (1.1) in the domain $D$ is specified by the formula

$2 U(x)=\int_{\partial D}\left(\Pi(y, x, \omega, \sigma)\left\{R\left(\partial_{y}, n(y)\right) U(y)\right\}-\right.$ 


$$
\left.-\left\{\tilde{R}\left(\partial_{y}, n(y)\right) \widetilde{\Pi}(y, x, \omega, \sigma)\right\}^{*} U(y)\right) d s_{y}, \quad x \in D,
$$

where $\Pi(y, x, \omega, \sigma)$ is the Carleman matrix and $\widetilde{\Pi}(y, x, \omega, \sigma)=\widetilde{\Psi}(y-x, \omega)+\widetilde{G}(y-x, \sigma)$.

Using this matrix, one can easily conclude the estimate stability of solution of the problem (1.1), (1.3) and also indicate effective method decision this problem as in [3], [4].

With a view to construct an approximate solution of the problem (1.1), (1.3) we construct the following matrix:

$$
\begin{aligned}
& \Pi(y, x, \omega, \sigma)=\llbracket \Pi_{k j}(y, x, \omega, \sigma) \rrbracket_{4 \times 4}, \\
& \Pi_{k j}(y, x, \omega, \sigma)=\sum_{l=1}^{3}\left[\left(1-\delta_{k 4}\right)\left(1-\delta_{j 4}\right)\left(\frac{\delta_{k j}}{2 \pi \mu} \delta_{3 l}-\alpha_{l} \frac{\partial^{2}}{\partial x_{k} \partial x_{j}}\right)+\right. \\
& \left.+\beta_{l}\left(i \omega \eta\left(1-\delta_{j 4}\right) \delta_{k 4} \frac{\partial}{\partial x_{j}}-\gamma\left(1-\delta_{k 4}\right) \delta_{j 4} \frac{\partial}{\partial x_{k}}\right)+\delta_{k 4} \delta_{j 4} \gamma_{l}\right] \Phi\left(y, x, \sigma, i \lambda_{l}\right), \quad k, j=1,2,3, \\
& \Pi_{4 j}(y, x, \omega, \sigma)=\sum_{l=1}^{3}\left\{i \beta_{l} \omega \eta\left(1-\delta_{j 4}\right) \frac{\partial}{\partial x_{j}}+\delta_{j 4} \gamma_{l}\right\} \Phi\left(y, x, \sigma, i \lambda_{l}\right), \quad j=1,2,3, \\
& \Pi_{k 4}(y, x, \omega, \sigma)=\sum_{l=1}^{3}\left\{-\beta_{l} \gamma\left(1-\delta_{k 4}\right) \frac{\partial}{\partial x_{k}}+\delta_{k 4} \gamma_{l}\right\} \Phi\left(y, x, \sigma, i \lambda_{l}\right), \quad k=1,2,3, \\
& \Pi_{44}(y, x, \omega, \sigma)=\sum_{l=1}^{3} \gamma_{l} \Phi\left(y, x, \sigma, i \lambda_{l}\right),
\end{aligned}
$$

Where

$$
\begin{aligned}
& \Phi(y, x, \sigma, \Lambda)=\frac{1}{-2 \pi^{2} \exp \left(\sigma x_{3}^{2}\right)} \int_{0}^{\infty} \operatorname{Im} \frac{\exp \left(\sigma w^{2}\right)}{w-x_{3}} \frac{\cos (\Lambda \Lambda u) d u}{\sqrt{u^{2}+\alpha^{2}}}, \\
& w=i \sqrt{u^{2}+\alpha^{2}}+y_{3}, \quad \alpha^{2}=\left(y_{1}-x_{1}\right)^{2}+\left(y_{2}-x_{2}\right)^{2}, \quad \alpha>0 .
\end{aligned}
$$

The following theorem was proved in [5].

Lemma2.1. For function $\Phi(y, x, \sigma, \Lambda)$, the following formula is valid

$$
\Phi(y, x, \sigma, i \Lambda)=\frac{\exp (i(i \Lambda r)}{4 \pi r}+\varphi(y, x, \sigma, \Lambda), r=|x-y| \text {, }
$$

where $\varphi(y, x, \sigma, \Lambda)-$ is a regular function that is defined for all $y$ and $x$ satisfies the Helmholtz equation: $\Delta\left(\partial_{y}\right) \varphi+\Lambda^{2} \varphi=0, y \in D, \Lambda^{2}>0$.

Moreover, for function $\Phi(y, x, \sigma, i \Lambda)$ holds following inequality

$\int_{\partial D \backslash S}\left(|\Phi(y, x, \sigma, i \Lambda)|+\left|\frac{\partial \Phi(y, x, \sigma, i \Lambda)}{\partial n}\right|\right) d s_{y} \leq C(\Lambda, D) \sigma \exp \left(-\sigma x_{3}^{2}\right)$,

Where $C(\Lambda, D)$ certain bounded function independent of $\sigma$ and

$\Delta\left(\partial_{y}\right)=\frac{\partial^{2}}{\partial y_{1}^{2}}+\frac{\partial^{2}}{\partial y_{2}^{2}}+\frac{\partial^{2}}{\partial y_{3}^{2}}$.

The function $\Phi(y, x, \sigma, \Lambda)$ we shall call Carleman's functions for the Helmholtz equation. For her holds following inequalities:

$$
\begin{aligned}
& |\Phi(y, x, \sigma, i \Lambda)| \leq \mathrm{C}_{1} r^{-1} \exp \sigma\left(y_{3}^{2}-x_{3}^{2}\right), \\
& \left|\frac{\partial \Phi(y, x, \sigma, i \Lambda)}{\partial \mathrm{y}_{k}}\right| \leq \mathrm{C}_{2} r^{-2} \sigma \exp \sigma\left(y_{3}^{2}-x_{3}^{2}\right), k=1,2,3, \\
& \left|\frac{\partial^{2} \Phi(y, x, \sigma, i \Lambda)}{\partial \mathrm{y}_{k} \partial \mathrm{y}_{j}}\right| \leq C_{3} r^{-3} \sigma^{2} \exp \sigma\left(y_{3}^{2}-x_{3}^{2}\right), k, j=1,2,3,
\end{aligned}
$$

here $C_{k}=$ const $, k=1,2,3$. 
From Lemma 2.1 we obtain

Lemma2.2. The matrix $\Pi(y, x, \omega, \sigma)$ given by (2.3) and (2.5) is Carleman's matrix for problem (1.1), (1.3).

By using (2.3), (2.4) and inequalities (2.7) we obtain

$\int_{\partial D \backslash S}\left(|\Pi(y, x, \omega, \sigma)|+\left|R\left(\partial_{y}, n(y)\right) \Pi(y, x, \omega, \sigma)\right|\right) d s_{y} \leq C(D) \sigma^{2} \exp \left(-\sigma x_{3}^{2}\right)$,

where $C(D)$ is a bounded function inside of $D$.

Let us set

$$
\begin{aligned}
& 2 U_{\sigma}(x)=\int_{S}\left(\Pi(y, x, \omega, \sigma)\left\{R\left(\partial_{y}, n(y)\right) U(y)\right\}-\right. \\
& \left.-\left\{\tilde{R}\left(\partial_{y}, n(y)\right) \widetilde{\Pi}(y, x, \omega, \sigma)\right\}^{*} U(y)\right) d s_{y}, \quad x \in D .
\end{aligned}
$$

The following theorem holds.

Theorem2.2. Let $U(x)$ be a regular solution of the system (1.1) in $D$ such that

$$
|U(y)|+\left|R\left(\partial_{y}, n(y)\right) U(y)\right| \leq M, y \in \partial D \backslash S .
$$

Then for $\sigma \geq 1$ the following estimate is valid:

$\left|U(x)-U_{\sigma}(x)\right| \leq M C(x) \sigma^{2} \exp \left(-\sigma x_{3}^{2}\right)$,

Where

$C(x) \int_{\partial D} \frac{d s_{y}}{r^{2}}$.

Since, by formulas (2.2) and (2.9) we have

$$
\begin{aligned}
& \left|U(x)-U_{\sigma}(x)\right| \leq \frac{1}{2} \mid \int_{\partial D \backslash S}\left(\Pi(y, x, \omega, \sigma)\left\{R\left(\partial_{y}, n(y)\right) U(y)\right\}-\right. \\
& \left.-\left\{\tilde{R}\left(\partial_{y}, n(y)\right) \widetilde{\Pi}(y, x, \omega, \sigma)\right\}^{*} U(y)\right) d s_{y} \mid \leq \\
& \leq \frac{1}{2} \int_{\partial D \backslash S}\left(|\Pi(y, x, \omega, \sigma)|+\left|\left\{\tilde{R}\left(\partial_{y}, n(y)\right) \widetilde{\Pi}(y, x, \omega, \sigma)\right\}^{*}\right|\right)\left(|U(y)|+\left|R\left(\partial_{y}, n(y)\right) U(y)\right|\right) d s_{y} .
\end{aligned}
$$

Now on the basis of (2.8) and (2.10) we obtain the required estimate.

Corollary. Provided theorem we have the following equivalent formulas continue

$$
\begin{aligned}
& U(x)=\lim _{\sigma \rightarrow \infty} U_{\sigma}(x)=\frac{1}{2} \lim _{\sigma \rightarrow \infty} \int_{S}\left(\Pi(y, x, \omega, \sigma)\left\{R\left(\partial_{y}, n(y)\right) U(y)\right\}-\right. \\
& \left.-\left\{\tilde{R}\left(\partial_{y}, n(y)\right) \widetilde{\Pi}(y, x, \omega, \sigma)\right\}^{*} U(y)\right) d s_{y}, \quad x \in D, \\
& U(x)=\frac{1}{2} \int_{S}\left(\Pi(y, x, \omega)\left\{R\left(\partial_{y}, n(y)\right) U(y)\right\}-\right. \\
& \left.-\left\{\tilde{R}\left(\partial_{y}, n(y)\right) \widetilde{\Pi}(y, x, \omega)\right\}^{*} U(y)\right) d s_{y}+\frac{1}{2} \int_{0}^{\infty} Q(x, \omega, \sigma) d \sigma, \quad x \in D .
\end{aligned}
$$


Where

$Q(x, \omega, \sigma)=\int_{S}\left(P(y, x, \omega, \sigma)\left\{R\left(\partial_{y}, n(y)\right) U(y)\right\}-\right.$
$\left.-\left\{\tilde{R}\left(\partial_{y}, n(y)\right) \tilde{P}(y, x, \omega, \sigma)\right\}^{*} U(y)\right) d s_{y}, \quad x \in D$,
$P(y, x, \omega, \sigma)=\frac{\partial}{\partial \sigma} \Pi(y, x, \omega, \sigma)=\llbracket \frac{\partial}{\partial \sigma} \Pi_{k j}(y, x, \omega, \sigma) \rrbracket_{4 \times 4}$.

$\Pi(y, x, \omega)$ matrix constructed according to the formula (2.3) and (2.4) at

$\Phi(y, x, i \Lambda)=\frac{\exp (i \Lambda r)}{4 \pi r}$.

Equivalence formulas continuation (2.11) and (2.12) follows from the formula

$\lim _{\sigma \rightarrow \infty} U_{\sigma}(x)=\int_{0}^{\infty} \frac{d U_{\sigma}(x)}{d \sigma} d \sigma+U_{0}(x)$

Based on the continuation of the formula (2.11) and (2.12) we give solvability criterion the Cauchy problem (1.1), (1.3).

Theorem2.3. Let $S \in C^{2}, f \in C^{1}(S), g \in C(S)$. then, for problem (1.3) to be

Solvable, it is necessary and sufficient that

$\left|\int_{0}^{\infty} \partial_{x}^{p} Q(x, \omega, \sigma) d \sigma\right|<\infty,|p| \leq 2$,

Where $p-$ a multi-index, uniformly on any compact $K \subset D, x \in K$.

\section{REFERENCES}

[1] V. D. Kupradze, T. V. Burchuladze, T. G. Gegeliya, ot. ab. Three-Dimensional Problems of the Mathematical Theory of Elasticity and ... [in Russian], Nauka, Moscow,1976.

[2] M. M. Lavrent'ev. Some Ill-Posed Problems of Mathematical Physics [in Russian], Computer Center of the Siberian Division of the Russian Academy of Sciences, Novosibirck (1962) 92p.

[3] I. E. Niyozov, O. I. Makhmudov . The Cauchy problem for the system of equations of thermoelasticity in $\$ E^{\wedge}\{n\} \$$. IIUM Engineering Journal, Vol.15, N1, 2014.pp.81-90.

[4] O. I. Makhmudov, I. E. Niyozov. Regularization of a solution to the Cauchy Problem for the System of Thermoelasticity.Contemporary Mathematics.AMS, Primary V382, 2005,74F05, 35Q72.

[5] Sh. Ya. Yarmukhamedov. Cauchy problem for the Laplace equation. Dokl.Acad.Nauk SSSR [Soviet Math.Dokl.], V.357, No.3, p.320-323.(1997).

\section{AUTHOR'S BIOGRAPHY}

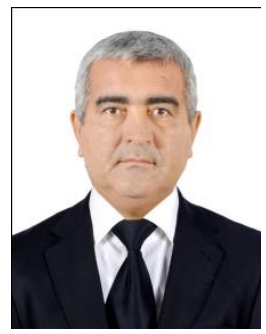

I. E. NIYOZOV, assistant professor, Department of Mechanics and Mathematics, Samarkand State, University,Uzbekistan

Citation: I. E. NIYOZOV, (2017). Solvability Criterion for the Cauchy Problem for the Theory of Elasticity, International Journal of Modern Studies in Mechanical Engineering (IJMSME), 3(4), pp.33-38, DOI: http://dx.doi.org/10.20431/2454-9711.0304004.

Copyright: () 2017 I. E. NIYOZOV. This is an open-access article distributed under the terms of the Creative Commons Attribution License, which permits unrestricted use, distribution, and reproduction in any medium, provided the original author and source are credited 